\title{
MODERATE ENANTIOSELECTIVITY PROMOTED BY CYCLODEXTRINS IN THE Zn-BARBIER ALLYLATION REACTION OF ALDEHYDES IN AQUEOUS MEDIUM
}

\author{
R. Fornasier ${ }^{1}$, F. Marcuzzi ${ }^{* 1}$ and D. Marton ${ }^{2}$ \\ 1 Department of Organic Chemistry and CNR Center of Organic Reaction Mechanism, \\ 2 Department of Inorganic, Metallorganic and Analytical Chemistry, \\ University of Padua, Marzolo Road 1, I-35131 Padua, Italy
}

\begin{abstract}
The effects exerted by native and trimethylated (-cyclodextrins in the $\mathrm{Zn}$-Barbier allylation reaction of aldehydes in aqueous solution has been studied. The rates of conversion were, generally, depressed in the presence of the cyclodextrins, especially TMCD, but the chemical yields of the products were very satisfactory, in all cases. Moderate enantioselectivity effects were also obtained in the presence of TMCD, whereas native (CD showed to be practically inactive. The results can be rationalised in terms of formation of inclusion complexes between the substrates and the CDs and of their interaction with the surface of the metal.
\end{abstract}

\section{Introduction}

Cyclodextrins (CDs) are a special class of natural chiral compounds, today available on the market at a reasonable price, which have found, in recent years, a number of useful applications ${ }^{1}$. They can be chemically modified in various ways in order to obtain derivatives with specific characteristics (e.g. trimethylated CD 2) ${ }^{2}$. We are particularly interested to investigate the feasibility of using cyclodextrins as chemical auxiliaries or catalysts to achieve stereoselective and enantioselective alkylations of carbonyl compounds with organometallic reagents. In previous works, we obtained promising results in the enantioselective addition reaction of diethylzinc to aldehydes, in the presence of alkylated -cyclodextrins, in organic solvents ${ }^{3}$ and in the allylation and $t$-butylation reactions of 2-cyclohexenone with the proper alkyl halides and $\mathrm{Zn}$ dust, in the presence of either native or methylated -cyclodextrins, in aqueous media ${ }^{4 a}$. The latter method, based on the protocol of the $\mathrm{Zn}$-Barbier reaction $4 b, c$, is particularly interesting since it offers the considerable advantage to work under aqueous conditions. As an extension of that work, we have applied the same method to the allylation of typical aldehydes, using allyl bromide and $\mathrm{Zn}$ dust, in the presence of native $C D 1$, or of the trimethylated derivative heptakis- $\left(2,3,6-0, O^{\prime}, O^{\prime \prime}\right.$-trimethyl)--cyclodextrin 2 (TMCD), as chiral auxiliaries.

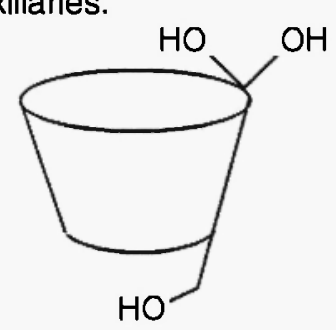

1

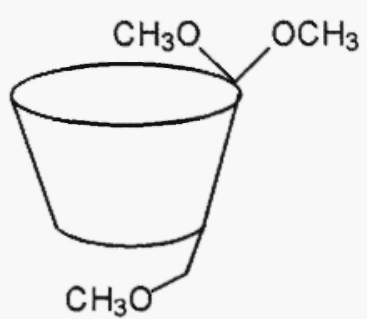

2

We wish to report here the results obtained.

\section{Materials and Methods}

All the starting materials were commercial products.

The experimental procedure adopted in this work was similar to that described in the protocol of the $\mathrm{Zn}$-Barbier reaction $4 \mathrm{~b}, \mathrm{c}$ The reaction products were analyzed and identified by standard techniques. All the spectroscopic data were in good agreement with the structures 9-13.

The enantiomeric excess (e.e.) of the mixtures of the reaction products was determined by Prof. F. Gasparrini at the Department of Study and Chemistry and Technology of Biologically Active Substances, University of Study "La Sapienza" (Rome), with a $(R, R)-N-D N B-D P E D A-C S P$ I chiral column $(250 \times 4.0 \mathrm{~mm})^{5}$. 


\section{Results and Discussion}

The reaction between the aldehydes 3-7 and allyl bromide 8, in the presence of $\mathrm{Zn}$ dust and cyclodextrins 1 or 2 , in saturated aqueous ammonium chloride solution, is schematically represented in the following equation.

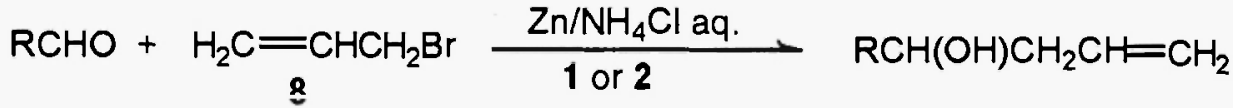

$$
\begin{aligned}
& \text { 3, } \mathrm{R}: \mathrm{PhCH}=\mathrm{CH} \quad(E) \\
& \text { 4, R: } \mathrm{PhCH}=\mathrm{C}\left(\mathrm{CH}_{3}\right)(\mathrm{Z}) \\
& \text { 5, R: } \mathrm{C}_{6} \mathrm{H}_{11} \\
& \text { 6, R : 1-naphthyl } \\
& \text { 7, } \mathrm{R}: \text { 2-naphthyl }
\end{aligned}
$$

$$
\text { 9, } \mathrm{R}: \mathrm{PhCH}=\mathrm{CH}
$$

\begin{tabular}{|c|c|c|c|c|c|c|c|}
\hline Entry & $\begin{array}{l}\text { Aldehyde } \\
\text { (1 mmole) }\end{array}$ & $\begin{array}{c}\mathbf{8} \\
\text { (mmole) }\end{array}$ & $\begin{array}{c}\mathrm{Zn} \\
\text { (mmole) }\end{array}$ & $\begin{array}{c}\mathrm{CD} \\
\text { (1 mmole) }\end{array}$ & $\begin{array}{l}\text { Time } \\
\text { (h) }\end{array}$ & $\begin{array}{c}\text { Conversion } \\
\%^{\mathrm{a}}\end{array}$ & $\begin{array}{c}\text { Alcohols }^{b} \\
\text { yield\%, }(e . e)^{c}\end{array}$ \\
\hline 1 & 3 & 3.0 & 2.4 & - & 1.0 & 90 & 9,96 \\
\hline 2 & 3 & 3.0 & 2.4 & 1 & 5.0 & 77 & 9,84 \\
\hline 3 & 3 & 6.0 & 4.8 & 1 & 1.3 & 84 & 9,88 \\
\hline 4 & 3 & 3.0 & 2.4 & 2 & 3.0 & 100 & $9.59,(3.0)$ \\
\hline 5 & 4 & 3.0 & 2.4 & - & 0.5 & 76 & 10,95 \\
\hline 6 & 4 & 6.0 & 4.8 & $=$ & 0.5 & 100 & 10,96 \\
\hline 7 & 4 & 3.0 & 2.4 & 1 & 1.0 & 12 & 10,90 \\
\hline 8 & 4 & 6.0 & 4.8 & 1 & 6 & 32 & 10,93 \\
\hline 9 & 4 & 3.0 & 2.4 & 2 & 3.0 & 93 & $10,98,(3.5)$ \\
\hline 10 & 5 & 3.0 & 2.4 & - & 0.16 & 100 & 11. 100 \\
\hline 11 & 5 & 3.0 & 2.4 & 1 & 20 & 90 & 11,47 \\
\hline 12 & 5 & 6.0 & 4.8 & 1 & 1.25 & 98 & 11,85 \\
\hline 13 & 5 & 3.0 & 2.4 & 2 & 3.0 & 78 & 11,100 \\
\hline 14 & 6 & 3.0 & 2.4 & $=$ & 1.0 & 50 & 12,86 \\
\hline 15 & 6 & 3.0 & 2.4 & 1 & 5.5 & 18 & 12,85 \\
\hline 16 & 6 & 6.0 & 4.8 & 1 & 20 & 42 & 12,91 \\
\hline 17 & 6 & 12.0 & 9.6 & 1 & 3.5 & 64 & 12,70 \\
\hline 18 & 6 & 3.0 & 2.4 & 2 & 3.0 & 91 & $12,99,(10)$ \\
\hline 19 & 7 & 3.0 & 2.4 & $=$ & 2.0 & 40 & 13,100 \\
\hline 20 & 7 & 6.0 & 4.8 & - & 1.0 & 97 & 13,100 \\
\hline 21 & 7 & 6.0 & 4.8 & 1 & 3.5 & 25 & 13,66 \\
\hline 22 & 7 & 3.0 & 2.4 & 2 & 3.0 & 32 & $13,100,(12)$ \\
\hline
\end{tabular}

10, $\mathrm{R}: \mathrm{PhCH}=\mathrm{C}\left(\mathrm{CH}_{3}\right)$

11, R: $\mathrm{C}_{6} \mathrm{H}_{11}$

12, R : 1-naphthyl

13, R : 2-naphthyl

Table. Results of the allylation reactions of aldehydes 3-7

aDetermined by G.C. ${ }^{D}$ Based on aldehyde converted. ${ }^{C} e . e$. determined by HPLC with a chiral column.

The results obtained, reported in the Table, deserve a brief comment. (i) In the absence of cyclodextrins, the conversion percentages range from good $(40 \%$, entry 19$)$ to excellent $(100 \%$, entry 10 ) and the reaction times are, generally, satisfactory; the cyclohexyl aldehyde, 5 , reacts much faster than all the other aldehydes, especially those derived from naphthalene, $\mathbf{6}$ and 7 , which appear to be the least reactive among the substrates tested in this preliminary study. (ii) The presence of the cyclodextrins affects significantly the conversion rate which is, generally, depressed. This effect is moderate in the presence of TMCD, but it is greatly enhanced in the presence of native $C D$; in the latter case, the difficulty can be partly circumvented by increasing the molar ratio of the reagents (see Table). (iii) The chemical yields of the alcohols obtained are, generally, very satisfactory. In all cases, the work-up of the reaction mixture and the isolation of the pure alcohols was an easy task. (iv) As far as the enantioselectivities observed in the reactions 
carried out in the presence of the two types of cyclodextrins, the results obtained were quite clear: while native $C D$ showed to be inactive in all cases, since the alcohols isolated were considered racemic, (at least in the limits of the sensitivity of the instruments used for the determination of the optical activity and of the enantiomeric enrichment), TMCD proved to be able to promote sizeable enantiomeric enrichments (3-12\%) in the reaction products (see entries $4,9,18$ and 22 in the Table).

The e.e. obtained is, indeed, rather modest; however, there are not so many examples of asymmetric inductions obtained with the $\mathrm{Zn}$-Barbier reaction reported in the literature $\mathrm{Ab}^{\mathrm{b}} \mathrm{c}$, and even though these results are inferior to those previously obtained by us with 2-cyclohexenone ${ }^{4 a}$, we think that they are significant, at least for the evaluation of the scope and limitations of this method.

Little is known about the mechanism of the $\mathrm{Zn}$-Barbier reaction in aqueous medium. According to recent hypotheses 6,7 , the reaction is believed to occur via formation of ion-radical species at the surface of the metal, where the reactants are adsorbed. This appears to be consistent with the decreasing of the conversion rates generally observed in all our reactions in the presence of both native and trimethylated CDs, since the inclusion of the substrate into the macrocycle should hinder its adsorption at the surface of the metal, where the reaction takes place. On the other hand, it is known that, in general, the inclusion complexes of hydrophobic organic substrates in native cyclodextrins are less soluble in water than the corresponding complexes in methylated cyclodextrins (TMCD); in fact, the former frequently precipitate as crystalline or amorphous powders, in contrast with the latter, which normally remain in solution. This behaviour was observed also in our reactions and, therefore, it seems reasonable that higher conversion rates and enantioselectivities could have been obtained in the reactions carried out in the presence of TMCD than in those in which native $C D$ was used. (It is worthy to remind here that also in the allylation of cyclohexenone TMCD proved to be a better chiral auxiliary than $C D^{4 a}$ ). As a matter of fact, in these reactions, since the highest e.e. has been obtained with those aldehydes, 6 and 7 , which, bearing the widest hydrophobic structure are, presumably, better included into the hydrophobic cavity of TMCD, a simple medium effect, similar to that exerted by a phase-transfer catalyst, can, probably, be excluded. It is not clear, however, whether the observed effects of the cyclodextrins in this type of reactions are to be ascribed to exclusive inclusion, into the cavity of the macrocycles, of the substrate, or also of the alkylating agent, or of some radical intermediate generated at the surface of the metal.

\section{Acknowledgment.}

Financial support by CNR and MURST (Rome) is gratefully acknowledged.

\section{References}

1. (a) Sanger, W. Anoew. Chem., Int. Ed. Engl., 1980, 19, 344. (b) Jones, S. P.; Grant, J. W.; Hadgraft, J.; Parr, G. D. Acta Pharm. Technol., 1984, 30, 213. (c) Szejtli, J." "Cyclodextrin Technology", Kluwer Ácademic Publisher, Dordrecht, 1988.

2. Fornasier, R.; Mammi, S.; Marcuzzi, F.; Morandin, M.; Moro, S. Gazz. Chim. Ital., 1997, 127, 63 and references cited therein.

3. Bonetto, L.; Fornasier, R.; Tonellato, U. Gazz. Chim. Ital., 1995, 125, 63.

4. (a) Fornasier, R.; Marcuzżi, F.; Piva, M.; Tonellato, U., Gazz. Chim. Ital.,126, 633 and references cited therein; '(b)'Blomberg, C. "The Zn-Barbier Reaction and Related"One-Step Processes" in Reactivity and Structure Concepts in Organic Chemistry, 31, Hafner K. et al Edirors, Springer Verlag, Berlin, 1993; (c) Yamamoto, Y.; Asao, N. Chem. Rev., 1993, 93, 2207.

5. Maier, N.M.; Uray G., Chirality, 1996, 8, 490.

6. Luche, J. L.; Allavena, C.; Petrier, C.; Dupuy, C. Tetrahedron Lett., 1988, 29, 5373.

7. Marton, D.; Stivanello, D.; Tagliavini, G. J. Org. Chem., 1996, 61, 2731.

\section{Received: November 19, 1997 - Accepted: November 27, 1997 - Accepted in revised camera-ready format: December 17, 1997}

